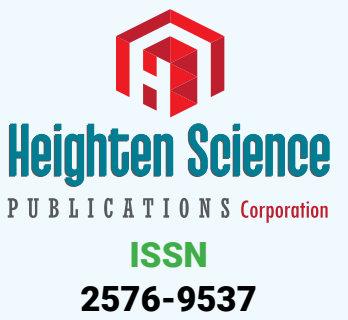

*Address for Correspondence: Tiziana Ciarambino, MD, PhD, Department of Internal Medicine, University of Campania, Hospita Marcianise, Caserta Italy, Tel: 3200639635 ; +39.081.566.6037

Email: tiziana.ciarambino@gmail.com

Submitted: 15 April 2019

Approved: 06 May 2019

Published: 07 May 2019

Copyright: (c) 2019 Laviscio E, et al. This is an open access article distributed under the Creative Commons Attribution License, which permits unrestricted use, distribution, and reproduction in any medium, provided the original work is properly cited

Keywords: Woman; Surgery; Abdominal hernia

Check for updates

\title{
A successful case report in woman: A gender medicine?
}

\author{
Emilio Laviscio ${ }^{1}$, Tiziana Ciarambino ${ }^{2 *}$, Annita Imbriani ${ }^{1}$, \\ Mauro Giordano ${ }^{2}$ and Filippo Topo ${ }^{1}$ \\ ${ }^{1}$ Department of Surgery, Hospital Marcianise, Caserta, Italy \\ ${ }^{2}$ Department of Internal Medicine, Hospital Marcianise, Caserta, Italy
}

\section{Abstract}

Introduction: Abdominal hernia is a pathological condition resulting from abnormal protrusion of abdominal viscera. In particular, internal hernias $(\mathrm{IH})$ represents about $0.2-0.9 \%$ of all cases with para- duodenal hernias while obturator hernias accounting for only $0.07 \%$ of all hernias.

Methods: We reported the case report of 79 year old women who was admitted to Internal Medicine Department of our Hospital for lung failure and after few days transferred to our Surgery Department for abdominal pain.

Conclusion: Obturator hernia is rare type of hernia and it is more frequent in older women with history of multiple pregnancy, chronic cough, and habitual constipation. In our patients, detailed physical examination and MRI preoperative imaging studies, have induce to the successful diagnosis.

\section{Introduction}

The obturator hernia is rare and is observed in older women, with a major frequency in the Chinese races [1-3]. Commonly, patients present with symptoms of intestinal obstruction [4-7].

\section{Case Report}

We reported the case of 79 year old women who was admitted to Internal Medicine Department of our Hospital for lung failure and after few days transferred to our Surgery Department for abdominal pain. She was affected by hypertension, COPD, thyroiditis and right inguinal hernia. Sin the previously week she complained coughing and dyspnea. After 3 hours post admission, she complains diffused abdominal pain, loss of appetite and nausea. The abdomen examination showed irreducible swelling in right inguinal region, and also completely closed. Laboratory Analysis demonstrated neutrophilic leukocytosis, increased mioglobin and LDH. The abdomen X-Rays showed disorderly distribution of intestinal bloating. The chest X-Rays showed atelectasis in the left basal area. The MRI studies demonstrated a slightly higher density. We determined that obturator hernia was associated with inflammatory exudates, and emergency surgery was performed. We removed massive obturator hernia strangulated and we proceeded to resection of omentum. Intestinal segment is incarcerated in the right obturator from the ileocecal part for $41 \mathrm{~cm}$. cutting the skin, it is darker gray, muddy, with the smell of liquid effluent (Figure 1). We proceeded to remove abscess and we performed plastic pug of the obturator foramen and control of hemostasis. The postoperative course was complicated by hematuria and dyspnoea. The patient was monitored by cardiorespiratory parameters and she was discharged on thirteenth day with medical therapy and outpatient controls. 


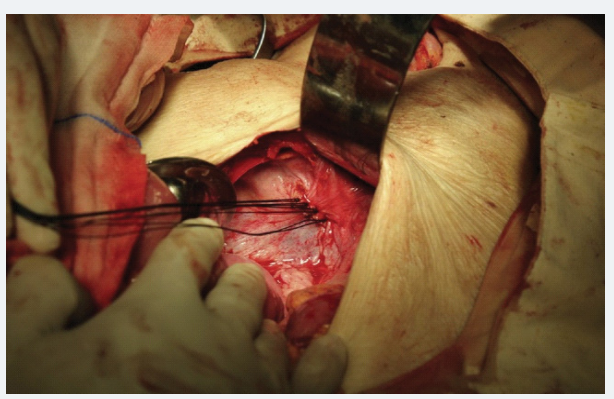

Figure 1: In this figure we reported the incarceration of the intestine. It was dark purple, like the film, in complete necrosis.

\section{Discussion}

The diagnosis is often delayed [8]. Strangulation is frequent and mortality remains high [9]. Pelvic obturator hernia is rare type of hernia [5] and it is more frequent in older women [10]. The late and difficult diagnosis makes this insidious disease. Thus, any delay in a diagnosis may lead to necrosis and perforation of the small bowel or a concomitant abscess. The only treatment for obturator hernias is surgery. An obturator hernia with a thigh abscess is extremely rare in Italy. In our case, resection of the perforated section of the small bowel and drainage of the abscess are both performed in a single surgical procedure. Often, the presence of symptoms that are obvious can be related to complications of other hernias. Accessory symptoms can be represented by pain in the nerve distribution area obturator with knee irradiation and the attitude in flexion and external rotation of the leg. Crucial is the role of ultrasound examination of the inguinal region and the knowledge of the anatomy of the obturator canal for proper therapeutic setting. The gold standard for diagnosis of certainty is confirmed by TC or MRI.

In our patients, detailed physical examination and MRI preoperative imaging studies, have induce to the successful diagnosis.

\section{References}

1. Martin LC, Merkle EM, Thompson WM. Review of internal hernias: radiographic and clinical findings. Am J Roentgenol. 2006; 186: 703-717. Ref.: https://tinyurl.com/y2hpmqz3

2. Akyildiz $H$, Artis $T$, Sozuer $E$, Akcan A, Kucuk $C$, et al. Internal hernia: complex diagnostic and therapeutic problem. Int J Surg. 2009; 7: 334-337. Ref.: https://tinyurl.com/y3pplty9

3. Mandarry MT, Zeng SB, Wei ZQ, Zhang C, Wang ZW. Obturator hernia - A condition seldom thought of and hence seldom sought. Int J Colorectal Dis. 2012; 27: 133-141. Ref.: https://tinyurl.com/y22m5pur

4. Lynch NP, Corrigan MA, Kearney DE. Andrews EJ. Successful laparoscopic management of an incarcerated obturator hernia. J Surg Case Rep. 2013; 2013: pii: rjt050. Ref.: https://tinyurl.com/yxdtfxcc

5. Bergstein JM, Condon RE. Obturator hernia: current diagnosis and treatment. Surgery. 1996; 119: 133-136. Ref.: https://tinyurl.com/y4udgkjb

6. Kazzazi A, Djavan B. Current status of pelvic lymph node dissection in prostate cancer: the New York PLND nomogram. Can J Urol. 2011; 18: 5585-5591. Ref.: https://tinyurl.com/y3p5z2hb

7. Mahdi B, Selim S, Hassen T, Mongi MM, Fadhel CM, et al. A rare cause of proximal intestinal obstruction in adults-annular pancreas: a case report. Pan Afr Med J. 2011; 10: 56. Ref.: https://tinyurl.com/y3ueo5ra

8. Munoz-Forner E, Garcia-Botello S, Lopez-Mozos F, Marti-Obiol R, Martinez-Lloret A, et al. Computed tomographic diagnosis of obturator hernia and its surgical management: A case series. Int $\mathrm{J}$ Surg. 2007; 5: 139-142. Ref.: https://tinyurl.com/y26bw2k8

9. Chan KV, Chan CK, Yau KW, Cheung MT. Surgical morbidity and mortality in obturator hernia: A 10-year retrospective risk factor evaluation. Hernia. 2014; 18: 387-392. Ref.: https://tinyurl.com/y5k5d65r

10. Skandalakis LJ, Androulakis J, Colborn GL, Skandalakis JE. Obturator hernia embryology, anatomy, and surgical applications. Surg Clin North Am. 2000; 80: 71-84. Ref.: https://tinyurl.com/yxkx8umy 